\title{
Using matrix factorisation for the prediction of electrical quantities
}

\author{
Fabian Lecron ${ }^{1 凶}$, Zacharie De Grève ${ }^{1}$, François Vallée ${ }^{1}$, Gerard Mor ${ }^{2}$, \\ Daniel Pérez², Stoyan Danov', Jordi Cipriano² \\ ${ }^{1}$ University of Mons, (UMONS), Faculty of Engineering, Mons, Belgium \\ ${ }^{2}$ Universitat Politècnica de Catalunya (UPC), International Centre for Numerical Methods in \\ Engineering (CIMNE), Terrassa, Spain \\ $\bowtie$ E-mail: fabian.lecron@umons.ac.be
}

\begin{abstract}
The prediction task is attracting more and more attention among the power system community. Accurate predictions of electrical quantities up to a few hours ahead (e.g. renewable production, electrical load etc.) are for instance crucial for distribution system operators to operate their network in the presence of a high share of renewables, or for energy producers to maximise their profits by optimising their portfolio management. In the literature, statistical approaches are usually proposed to predict electrical quantities. In the present study, the authors present a novel method based on matrix factorisation. The authors' approach is inspired by the literature on data mining and knowledge discovery and the methodologies involved in recommender systems. The idea is to transpose the problem of predicting ratings in a recommender system to a problem of forecasting electrical quantities in a power system. Preliminary results on a real wind speed dataset tend to show that the matrix factorisation model provides similar results than auto regressive integrated models in terms of accuracy (MAE and RMSE). The authors' approach is nevertheless highly scalable and can deal with noisy data (e.g. missing data).
\end{abstract}

\section{Introduction}

The prediction task is attracting more and more attention among the power system community. Accurate predictions of electrical quantities up to a few hours ahead (e.g. renewable production, electrical load etc.) are for instance crucial for distribution system operators to operate their network in the presence of a high share of renewables, or for energy producers to maximise their profits by optimising their portfolio management. Even the importance of predicting unknown data in the past has been noted in [1].

In the literature, statistical approaches are usually proposed to predict electrical quantities such as electrical load or wind speed data (in relation with the prediction of renewable production). First predictive models were auto regressive, auto regressive moving average (ARMA) and auto regressive integrated (ARIMA) models [2]. For instance, a periodic autoregressive model was used in [3] to perform customer segmentation based on the forecast of short-term load. Wind speed data were also predicted by ARMA models in [4].

Other methods involving machine-learning techniques can be identified. In this context, artificial neural networks have been extensively used in the literature [2, 5-8]. Other specific approaches relying on generalised principal component analysis [9] or extreme learning machines [10] were also proposed.

In the present paper, we introduce a novel method based on matrix factorisation to predict electrical quantities. Our approach is inspired by the literature on data mining and knowledge discovery and the methodologies involved in recommender systems.

Recommender systems are powerful tools that provide people with recommendations of items they will appreciate, based on their past preferences, history of purchase etc. A standard way to perform this task is to consider a matrix $\boldsymbol{X}$ of ratings where each row stands for a user and each column for an item (e.g. a movie). An element $x_{i j}$ of the matrix is a rating given by the user $i$ to the item $j$. The objective is to predict the unknown $x_{i j}$ 's in the matrix $\boldsymbol{X}$. One of the most effective techniques to do so is matrix factorisation (MF). It has been popularised by the winners of the Netflix prize in [11]. The idea is to decompose the incomplete matrix $\boldsymbol{X}$ into the product of two matrices $\boldsymbol{W}$ and $\boldsymbol{H}$. Finally, the unknown values in the matrix $X$ can be obtained by computing $\boldsymbol{W H}$.

Our contribution is to propose a MF method to forecast electrical quantities by transposing the problem of predicting ratings in a recommender system to a problem of forecasting (e.g. electrical load or wind speed for estimating renewable production). As a consequence, each row in the matrix $\boldsymbol{X}$ stands for a given period of time (a day, a week etc.) and each column stands for the temporal granularity of the data (quarter-hourly data, hourly data etc.). To illustrate our purpose, let us consider wind speed data (which can be transformed into the power produced by a wind farm using a power curve). The matrix $\boldsymbol{X}$ is first built with previous measurements of wind speed. Then, the last row is filled with the last known measurements of the current day. As a consequence, the last columns of the last row are unknown and can be forecasted by factorising the matrix.

A very few works are dedicated to the prediction of electrical quantities based on MF. Authors in [12] used sparse MF for that matter. Very recently, temporal dependency was included into a regularised MF procedure [13]. The interest of these approaches is to be highly scalable and to deal with noisy data (e.g. missing data).

In this work, a real dataset of wind speed data was extracted from the Royal Netherlands Meteorological Institute database. The prediction accuracy was evaluated by computing the mean absolute error (MAE) and the root mean square error (RMSE). The method has been compared with the persistence model and ARIMA prediction models. First results tend to show that the MF model provides similar results than ARIMA models in terms of accuracy (MAE and RMSE).

\section{Methods}

\subsection{Matrix factorisation}

MF allows us to decompose a given matrix into the product of two matrices. 
It can be proven [14] that the rank factorisation of a matrix $\boldsymbol{X} \in \mathbb{R}^{m \times n}$ of rank $f$ is a factorisation:

$$
\boldsymbol{X}=\boldsymbol{W H},
$$

where $\quad \boldsymbol{W} \in \mathbb{R}^{m \times f}, \boldsymbol{H} \in \mathbb{R}^{f \times n}, \quad$ and $\quad \operatorname{rank}(\boldsymbol{X})=\operatorname{rank}(\boldsymbol{W})=$ $\operatorname{rank}(\boldsymbol{H})=f$.

Based on that, we can define the low-rank matrix approximation of a matrix $\boldsymbol{X} \in \mathbb{R}^{m \times n}$. The idea is to find the best approximation $\widehat{\boldsymbol{X}} \simeq \boldsymbol{X}$ such that $\operatorname{rank}(\widehat{\boldsymbol{X}}) \leq f$. This problem can be written as [15]

$$
\min _{\widehat{\boldsymbol{X}}}\|\boldsymbol{X}-\widehat{\boldsymbol{X}}\|_{F}^{2} \quad \text { such that } \operatorname{rank}(\widehat{\boldsymbol{X}}) \leq f
$$

where \|\|$_{F}$ is the Frobenius norm.

Since rank $(\widehat{\boldsymbol{X}}) \leq f, \widehat{\boldsymbol{X}}$ can be factorised following (1). The problem defined in (2) becomes

$$
\min _{\mathbf{W}, \mathbf{H}}\|\boldsymbol{X}-\boldsymbol{W H}\|_{F}^{2},
$$

where $\widehat{\boldsymbol{X}}=\boldsymbol{W H}$.

\subsection{MF for missing data}

When data are missing in the matrix $\boldsymbol{X}$, it is possible to approximate $X$ and then recovers the missing data. In the collaborative filtering community, MF techniques are widely used to recover missing data, for instance to build recommen

\section{Will -irst introduce recommender sy}

are recovered and then transpose the

prediction of electrical quantities.

Recommender systems try
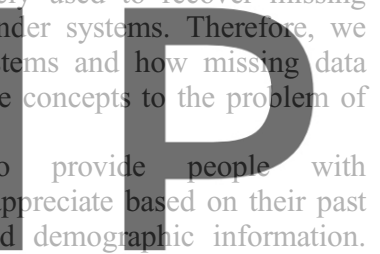

recommendations of items they will
preferences, history of purchase,

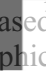

on their past

in most standard recommender systems are often represented with a matrix since it is characterised by three sets: a set of users

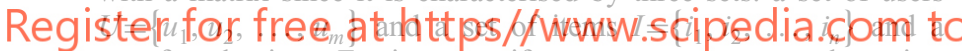

set of evaluations. For instance, if we want to recommend movies, evaluation could be a rating $r_{u i}$ given by a user $u$ to a movie $i$. Therefore, a $m \times n$ matrix $R$ is buitt with all the known ratings. By predicting the unknown ratings, one can know the predicted interest of one user for an item and make recommendations accordingly. Let us consider a matrix $\boldsymbol{R}$ with three users and three movies. User 1 has seen movie 2 , user 2 has seen movie 3 , and user 3 has seen movie 1. The matrix $\boldsymbol{R}$ would be

$$
R=\left(\begin{array}{lll}
? & r_{u_{1} i_{2}} & ? \\
? & ? & r_{u_{2} i_{3}} \\
r_{u_{3} i_{1}} & ? & ?
\end{array}\right)
$$

where '?' are unknown ratings.

In [11], Koren et al. present a basic MF model based on (3). With this technique, a rating $r_{u i}$ can be approximated by $\hat{r}_{u i}$, the inner product of user-item interactions. If we consider matrices $\boldsymbol{W}$ and $\boldsymbol{H}$ described in (3), we can write that

$$
\hat{r}_{u i}=w_{u} h_{i}
$$

where $w_{u}$ is a row of $\boldsymbol{W}$ representing user $u$ and $h_{i}$ is a column of $\boldsymbol{H}$ representing item $i$.

The challenge is then to compute all the $w_{u}$ and $h_{i}$. For that matter, it is described in [11] that $w_{u}$ and $h_{i}$ can be obtained by modelling directly the known ratings only, while avoiding over fitting thanks to a regularised model. As a consequence, the system proposed in [11] will perform a MF by only considering known ratings and the objective function to be minimised is written as

$$
\min _{\boldsymbol{w} *, \boldsymbol{h} *} \sum_{(u, i) \in \kappa}\left(r_{u i}-w_{u} h_{i}\right)^{2}+\lambda\left(\left\|w_{u}\right\|^{2}+\left\|h_{i}\right\|^{2}\right)
$$

where $\kappa$ is the set of the $(u, i)$ pairs for which $r_{u i}$ is known, $\lambda$ is a parameter controlling the regularisation to avoid over fitting.

Finally, computing all the $w_{u}$ and $h_{i}$ allows to obtain the matrices $\boldsymbol{W}$ and $\boldsymbol{H}$. The product between $\boldsymbol{W}$ and $\boldsymbol{H}$ then gives an approximation of matrix $\boldsymbol{R}$

Problem defined in (6) can be solved with stochastic gradient descent. Further details on how to implement it are provided in [11].

\subsection{Prediction of electrical quantities}

To transpose the problem of recommender systems to the problem of predicting electrical quantities, we first have to adapt the data. For recommender systems, we had a matrix with $r_{u i}$, a rating given by a user to an item. Now, we have an electrical quantity (e.g. electrical load), a period of time (e.g. a day, a week etc.) and a temporal granularity of the data (e.g. quarter-hourly data, hourly data etc.).

Let us imagine that today is Saturday and that we want to predict the electrical load of one user for the next day, i.e. Sunday. Furthermore, we have recorded the electrical load corresponding to this user for the last three weeks. As a consequence, we have a small matrix with three rows (the last row is corresponding to the current week) and seven columns (column 1 is Monday and column 7 is Sunday). We know all the $r_{u i}$ in the matrix, except the one at the intersection of the last row and the last column. Our
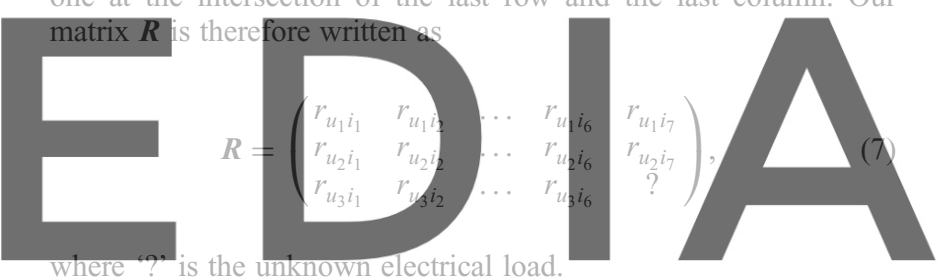

Actually, matrix in (7) has a similar structure to matrix in (4)

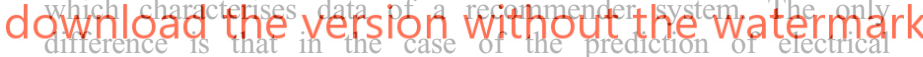

quantities, only one row and the last columns are concerned by unknown values, since the data to be predicted are in the future.

As a consequence, the unknown electrical load of matrix $\boldsymbol{R}$ in (7) can be found with the factorisation of $\boldsymbol{R}$, following (6).

Finally, we provide an illustration of a specific behaviour when we predict electrical quantities with MF. If we consider the matrix in (7), $u_{3}$ is the current week, $i_{1}$ is Monday and $i_{7}$ is Sunday. As we proposed, let us consider that we are on Saturday and that we want to predict the electrical load for Sunday but also for the Monday of the next week. We could add a fourth row in our matrix but this row would be characterised by only unknown values. Therefore, we have to shift the data in the matrix in such a way that the Monday of the fourth week appears on the third row. Such a shift implies

$$
\boldsymbol{R}=\left(\begin{array}{ccccc}
r_{u_{1} i_{2}} & r_{u_{1} i_{3}} & \ldots & r_{u_{1} i_{7}} & r_{u_{2} i_{1}} \\
r_{u_{2} i_{3}} & r_{u_{2} i_{3}} & \ldots & r_{u_{2} i_{7}} & r_{u_{3} i_{1}} \\
r_{u_{3} i_{2}} & r_{u_{3} i_{3}} & \ldots & ? & ?
\end{array}\right)
$$

where '?' are the unknown electrical loads.

\section{Experiments}

\subsection{Data and methods}

In the present paper, we decided to perform experiments on wind speed data as a first application. 


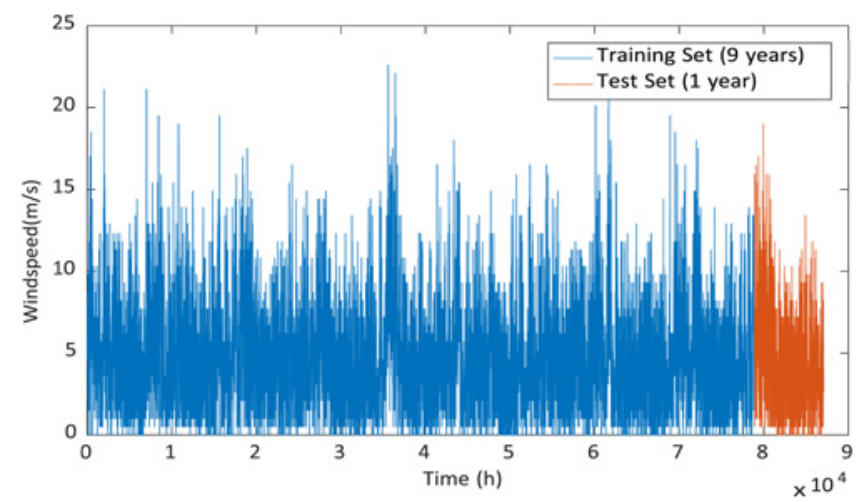

Fig. 1 Wind speed dataset used for the experiments

The dataset used in these experiments contains wind speeds measured in Rotterdam, the Netherlands, from 1986 to 1995. Data were obtained on the website of the Koninklijk Nederlands Meteorologisch Instituut (KNMI). The wind speeds were measured every hour. As a consequence, the resulting matrix $\boldsymbol{R}$ has 3652 rows and 24 columns. The dataset was divided into one training set containing the first 9 years of data (from 1986 to 1994). The last year (1995) was used as a test set (Fig. 1). The matrix for the training set $\left(\boldsymbol{R}_{\text {training }}\right)$ and the matrix for the test set $\left(\boldsymbol{R}_{\text {test }}\right)$ have the following form:
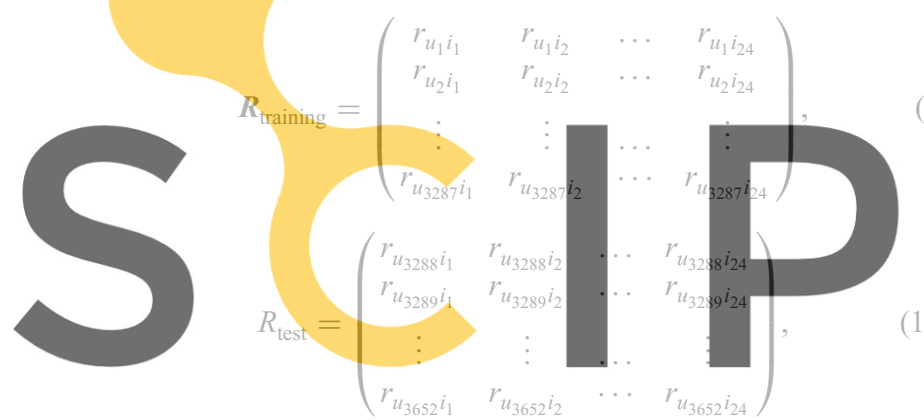

\section{Register for free at https//www.scipedia.com to} First, (6) is used to factorise matrix $\boldsymbol{R}_{\text {training, according to }}$

$$
\begin{gathered}
\boldsymbol{R}_{\text {training }}=\boldsymbol{W}_{\text {training }} \boldsymbol{H}_{\text {training }}, \\
\text { where } \quad \boldsymbol{W}_{\text {training }} \in \mathbb{R}^{3287 \times f} \text { and } \boldsymbol{H}_{\text {training }} \in \mathbb{R}^{f \times 24} \text {. }
\end{gathered}
$$

If we consider that we want to predict the 24th hour of the first day of 1995 (i.e. we want to predict $r_{u 3288 i_{24}}$ in matrix $\boldsymbol{R}_{\text {test }}$ ), we need to create a new matrix by adding the first row of matrix $\boldsymbol{R}_{\text {test }}$ to matrix $\boldsymbol{R}_{\text {training. }}$. We obtain

$$
\boldsymbol{R}_{\text {training, new }}=\left(\begin{array}{cccc}
r_{u_{1} i_{1}} & r_{u_{1} i_{2}} & \ldots & r_{u_{1} i_{24}} \\
r_{u_{2} i_{1}} & r_{u_{2} i_{2}} & \ldots & r_{u_{2} i_{24}} \\
\vdots & \vdots & \ldots & \vdots \\
r_{u_{3288} i_{1}} & r_{u_{3288} i_{2}} & \ldots & ?
\end{array}\right) \text {, }
$$

where '?' is the unknown wind speed we want to predict.

On this new matrix, we only need to use (6) to obtain the last row of the new matrix $\boldsymbol{W}$, which corresponds to the row we have added to $\boldsymbol{R}_{\text {training. }}$. This process is repeated for all the values contained in $\boldsymbol{R}_{\text {test }}$.

The approach described in the present work is mainly influenced by three parameters: the number of factors conditioning the rank of the approximated matrix $\boldsymbol{W H}$, the regularisation parameter $\lambda$ in the optimisation problem in (6) and the number of iterations needed by the gradient descent algorithm to converge to an adequate solution. The optimal value of these parameters has been calculated by performing an exhaustive search among values
Table 1 MAE and RMSE values for the persistence model

\begin{tabular}{lccccc}
\hline \multicolumn{1}{l}{ Persistence model } & & & & \\
\hline Horizon & $1 \mathrm{~h}$ & $2 \mathrm{~h}$ & $3 \mathrm{~h}$ & $4 \mathrm{~h}$ & $5 \mathrm{~h}$ \\
\hline MAE & 0.8061 & 1.0466 & 1.2405 & 1.4276 & 1.5960 \\
RMSE & 1.1445 & 1.4589 & 1.7074 & 1.9338 & 2.1376 \\
\hline
\end{tabular}

Table 2 MAE and RMSE values for the ARIMA model

\begin{tabular}{lccccc}
\hline ARIMA model & \multicolumn{7}{l}{} \\
\hline Horizon & $1 \mathrm{~h}$ & $2 \mathrm{~h}$ & $3 \mathrm{~h}$ & $4 \mathrm{~h}$ & $5 \mathrm{~h}$ \\
\hline MAE & 0.8041 & 1.0290 & 1.2081 & 1.3731 & 1.5162 \\
RMSE & 1.1077 & 1.4011 & 1.6240 & 1.8163 & 1.9799 \\
\hline
\end{tabular}

Table 3 MAE and RMSE values for our approach

\begin{tabular}{lccccc}
\hline Our approach & \multicolumn{6}{l}{} \\
\hline Horizon & $1 \mathrm{~h}$ & $2 \mathrm{~h}$ & $3 \mathrm{~h}$ & $4 \mathrm{~h}$ & $5 \mathrm{~h}$ \\
\hline MAE & 0.8954 & 1.0692 & 1.2197 & 1.3593 & 1.4844 \\
RMSE & 1.2155 & 1.4445 & 1.6361 & 1.8103 & 1.9687 \\
\hline
\end{tabular}

manually defined. The optimal values were chosen as the ones providing the lowest MAE resulting from the experiments.

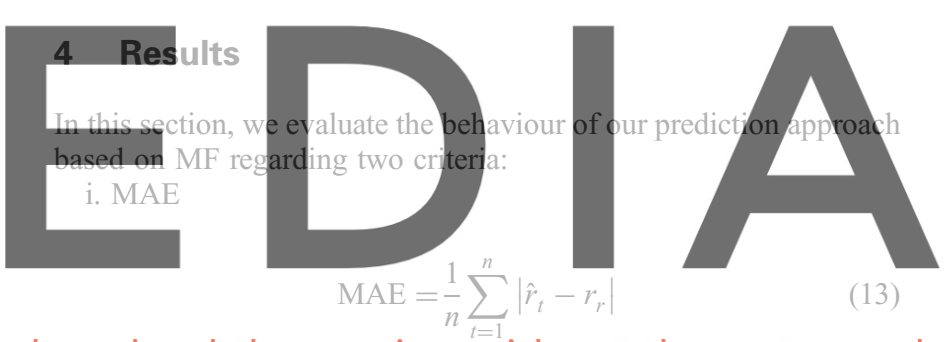
download the version without the watermark
ii. RMSE

$$
\mathrm{RMSE}=\sqrt{\frac{1}{n} \sum_{t=1}^{n}\left|\hat{r}_{t}-r_{r}\right|^{2}}
$$

In (13) and (14), $n$ is the number of values in the test set, $\hat{r}_{t}$ is a predicted value, and $r_{t}$ is the real value corresponding to $\hat{r}_{t}$.

Two methods were used in order to evaluate and compare our approach:

(i) Persistence model: it assumes that the future value to be predicted is equal to the last known value.

(ii) ARIMA model [16]: we used the ARIMA forecast tool in MATLAB to implement this approach.

The analysis of Tables 1-3 shows that at a short-time horizon $(1 \mathrm{~h})$, the ARIMA model is the best but the persistence model presents an interesting precision too. From a horizon time of $3 \mathrm{~h}$, our approach is very competitive with the ARIMA model in terms of accuracy (MAE and RMSE).

\section{Conclusion}

In this paper, we proposed a novel method based on MF to predict electrical quantities. Our approach is inspired by the literature on data mining and knowledge discovery and the methodologies involved in recommender systems. Our contribution was to 
propose a MF method by transposing the problem of predicting ratings in a recommender system to a problem of forecasting. Experiments were performed on a real wind speed dataset from the KNMI. Results showed that our approach is competitive with ARIMA models. However, an interesting characteristic of MF is its ability to be highly scalable and to deal with noisy data or with missing data. In the future, it would be interesting to evaluate the impact with larger datasets and with noisy data on the precision of prediction. Another interesting property of MF is the possibility to introduce a priori knowledge into the mathematical formulation (e.g. the results of an independent ARIMA forecast). By doing so, accuracy is therefore expected to improve, placing MF as a promising alternative to forecast electrical quantities.

\section{References}

1 Hocaog̃lu, F., Kurban, M.: 'The effect of missing wind speed data on wind power estimation'. Intell. Data Eng. Autom. Learn. - IDEAL 2007, 2007, 4881, pp. $107-114$

2 Ding, N., Benoit, C., Foggia, G., et al.: 'Neural network-based model design for short-term load forecast in distribution systems', IEEE Trans. Power Syst., 2016, 31, (1), pp. 72-81

3 Espinoza, M., Joye, C., Belmans, R., et al.: 'Short-term load forecasting, profile dentification, and customer segmentation: a methodology based on periodic time series', IEEE Trans. Power Syst., 2005, 20, (3), pp. 1622-1630

4 Erdem, E., Shi, J.: 'Arma based approaches for forecasting the tuple of wind speed and direction', Appl. Energy, 2011, 88, (4), pp. 1405-1414
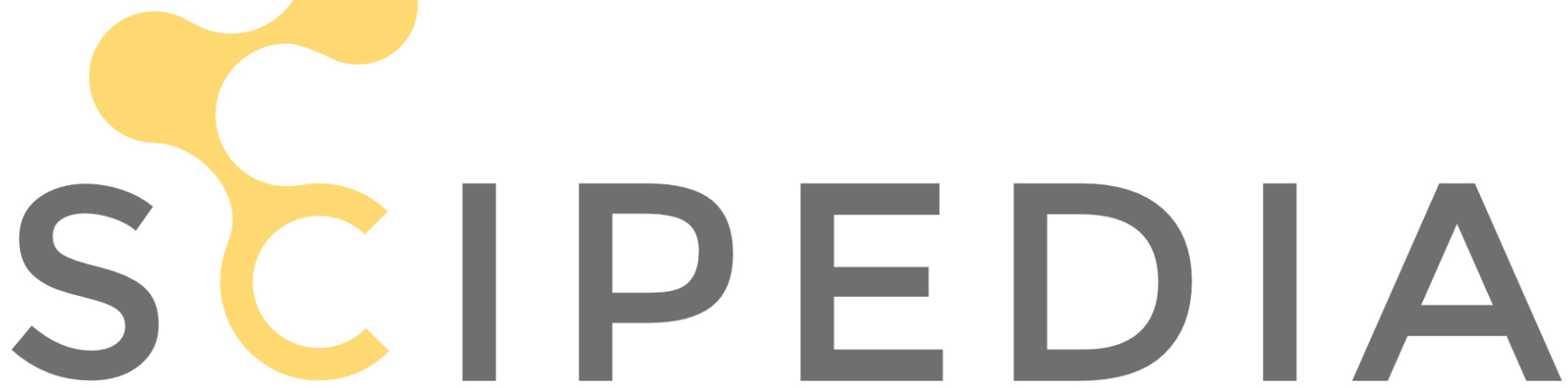

Register for free at https//www.scipedia.com to download the version without the watermark neural network', IEEE Trans. Power Syst., 2002 17, (1), pp. 113-118

Doucoure, B., Agbossou, K., Cardenas, A.: 'Time series prediction using artificial wavelet neural network and multi-resolution analysis: application to wind speed data', Renew. Energy, 2016, 92, pp. 202-211 distribution systems for proactive voltage cont
Distribution (CIRED 2015), 2015, pp. 1-5

Siripitayananon, P., Chen, H.-C., Jin, K.-R.: 'Estimating missing data of wind

Hu, Q., Su, P., Yu, D., et al: 'Pattern-based wind speed prediction based on

Ak, R., Fink, O., Zio, E.: 'Two machine learning approaches for short-term wind speed time-series prediction', IEEE Trans. Neural Netw. Learn. Syst., 2016, 27, (8), pp. $1734-1747$

recommender systems', Computer, 2009, 42, (8), pp. 30-37

2 Kim, S., Giannakis, G. B.: 'Load forecasting via low rank plus sparse matrix factorization'. 2013 Asilomar Conf. on Signals, Systems and Computers, Pacific Grove, CA, USA, November 3-6, 2013, 2013, pp. 1682-1686

3 Yu, H.-F., Rao, N., Dhillon, I. S.: 'Temporal regularized matrix factorization for U. V., Guyon, I., Garnett, R., (Ed.): 'Advances in neural information processing systems 29' (2016), pp. 847-855

4 Meyer, C. D.: 'Matrix analysis and applied linear algebra' (Society for Industrial and Applied Mathematics, 2000)

Gillis. N.: Non-negative matrix factorization: complexity, algorithms and

Box, G., Jenkins, G., Reinsel, G.: 'Time series analysis: forecasting and control' (Prentice-Hall, NJ, 1994, 3rd edn.)
Garcia, E., Canha, L., Pereira, P.: 'Hybrid forecasting techniques applied to speeds using neural network'. Southeast Con, 2002. Proc. IEEE, 2002, applications'. PhD thesis, Université catholique de Louvain, 2011 